\title{
Custo da produção de Orius insidiosus como agente de controle biológico
}

\author{
Simone Martins Mendes ${ }^{(1)}$,Vanda Helena Paes Bueno(1), Lívia Mendes Carvalho( ${ }^{(1)}$ \\ e Ricardo Pereira Reis ${ }^{(2)}$
}

\begin{abstract}
(1)Universidade Federal de Lavras (Ufla), Dep. de Entomologia, Caixa Postal 37, CEP 37200-000 Lavras, MG. E-mail: mmsimone@brfree.com.br, vhpbueno@ufla.br, carvalholm@hotmail.com (2)Ufla, Dep. de Administração e Economia. E-mail: ricpreis@ufla.br
\end{abstract}

\begin{abstract}
Resumo - O objetivo deste trabalho foi estimar o custo de produção por indivíduo do predador Orius insidiosus (Say), levando-se em consideração um modelo de criação em laboratório. Nos cálculos, considerou-se uma produção de 33.000 percevejos mês ${ }^{-1}$, dos quais 14,5\% foram para manutenção da criação e o restante, ou seja, 28.272 indivíduos, para comercialização. Os preços dos materiais e equipamentos utilizados na criação foram cotados em dólares americanos na proporção de R \$ 2,84 por US\$1,00. O custo estimado de cada percevejo predador foi da ordem de US\$ 0,069, levando-se em consideração custos fixos e variáveis. Este custo é um parâmetro decisivo na criação massal de Orius insidiosus e em sua utilização como agente de controle em programas de controle biológico de tripes.
\end{abstract}

Termos para indexação: controle biológico, criação massal, percevejo predador.

\section{Production cost of Orius insidiosus as biological control agent}

\begin{abstract}
The objective of this work was to estimate the individual production cost of the predator Orius insidiosus (Say) in laboratory. A rearing model of $O$. insidiosus in laboratory was used to reach production of 33,000 individuals month ${ }^{-1}$, using $14.5 \%$ to maintain the predator rearing in the laboratory and 28,272 individuals for commercialization. The prices of different materials and equipments used on predator rearing were fixed at US\$ $1.00=\mathrm{R} \$ 2.84$. The estimated cost for production of each adult predator was US\$ 0.069 , taking all the fixed and variable costs. This parameter is crucial to support $O$. insidiosus mass rearing, and to use this predator as biocontrol agent in biological control program of thrips.
\end{abstract}

Index terms: biological control, mass production, predator.

\section{Introdução}

A comercialização de inimigos naturais e o aumento de seu uso no Manejo Integrado de Pragas (MIP) são desafios para os entomologistas envolvidos com o controle biológico. Uma das respostas a este desafio é a redução do custo de criação destes agentes, por meio do aperfeiçoamento nas técnicas de criação e do aumento da eficácia dos agentes de controle biológico no campo (Tauber et al., 2000).

A utilização de inimigos naturais como agentes de controle biológico está intimamente ligada à sua produção em laboratório. Segundo Chambers (1977), a criação massal é a produção de insetos com aceitável relação custo/benefício. O estudo dos aspectos técnicos e econômicos da criação do inimigo natural é fundamental para o seu emprego efetivo como agente de controle biológico no campo ou em cultivos protegidos. De acordo com Lenteren (2000a), o desenvolvimento de técnicas de produção massal, controle de qualidade, armazenamento, envio e liberação de inimigos naturais pode levar à redução do custo de produção e à melhoria da qualidade do produto, viabilizando sua utilização.

A criação comercial economicamente viável de insetos entomófagos tem sido a meta de diversos programas de controle de pragas (Cohen et al., 1999). O predador Orius insidiosus (Say) vem sendo utilizado com sucesso no controle de tripes e ácaros em plantios comerciais de flores e hortaliças em cultivos protegidos e em programas de MIP, especialmente no Canadá, EUA e Europa (Lenteren, 2000b). Este predador é produzido por cerca de quatro empresas comerciais e vendido ao preço de U\$ 0,04 percevejo ${ }^{-1}$ (Lenteren et al., 1997; Bueno, 2000).

Os métodos de criação utilizados pelas empresas não são públicos, e por isso o estabelecimento de uma técni- 
ca de criação e do custo de produção é necessário quando se deseja a implementação deste método de controle biológico. A análise econômica permite se conhecer, em termos monetários, cada atividade da criação (Lopes \& Carvalho, 2000). Sua importância reside no fato de que a decisão sobre determinada tática de controle dentro do MIP depende do custo da sua implementação.

O objetivo deste trabalho foi estimar o custo de produção de Orius insidiosus, levando-se em consideração um modelo de criação para fins de pesquisa desse predador em laboratório, visando fornecer subsídios para sua comercialização e utilização como agente de controle biológico.

\section{Material e Métodos}

Na estimativa do custo de produção, utilizaram-se informações referentes à criação do predador Orius insidiosus, em recipientes de vidro de 1,7 L, na densidade de 250 ninfas recipiente ${ }^{-1}$ e 400 adultos recipiente $^{-1}$ (Figura 1).

O planejamento quantitativo da criação de $O$. insidiosus foi elaborado considerando-se os seguintes dados: um recipiente com 400 adultos do predador produz, em média, 62.400 ovos mês $^{-1}$; estes ovos são mantidos em 250 recipientes (potes de vidro com 1,7 L)

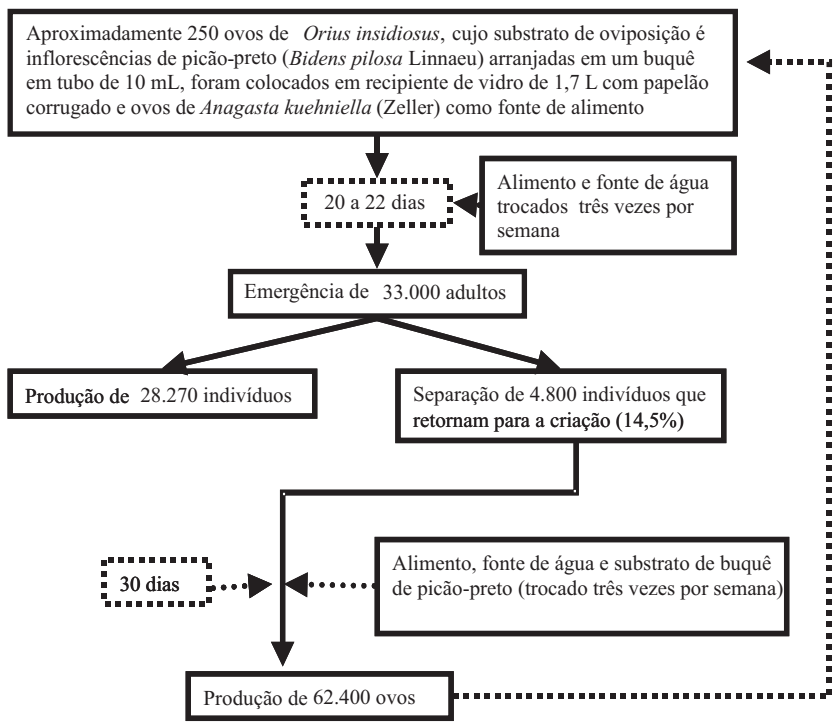

Figura 1. Fluxograma da criação de Orius insidiosus em sala climatizada a $26 \pm 2{ }^{\circ} \mathrm{C}$ e fotófase de 12 horas, de acordo com dados obtidos no esquema de criação adaptado de Bueno (2000). de criação; com este modelo, a viabilidade média é de $53 \%$, isto é, 33.000 adultos mês ${ }^{-1}$; do número total de indivíduos produzidos, 4.800 adultos retornam para a criação; dessa forma, este sistema produz em torno de 28.272 indivíduos mês ${ }^{-1}$ que podem ser comercializados ou liberados no campo (Figura 1).

O tempo de execução do modelo de criação consiste no período de 20 a 22 dias para a criação da fase imatura e de 30 dias para a coleta de ovos, além de um período para limpeza e higienização da criação e de um período como margem de segurança. Desta forma foram estabelecidos 60 dias para cada ciclo de produção do predador.

$\mathrm{Na}$ teoria de custo, conceitua-se custo total de produção como a soma dos valores dos recursos fixos e variáveis. É a soma de todos os recursos (insumos + capital fixo) e operações (mão-de-obra + taxas + serviços gerais) utilizados no processo produtivo da atividade, incluindo o respectivo custo alternativo ou de oportunidade, que, segundo Reis (2002), é a remuneração normal ao capital e trabalho alocados na atividade em questão.

O custo fixo (CF) corresponde ao custo dos recursos que têm duração superior a um ciclo de produção, sendo, nesse caso, o ciclo de produção do predador. É o custo de cada recurso fixo, que foi calculado mediante a soma de sua depreciação e seu custo alternativo.

A depreciação é o custo necessário para substituir os bens de capital quando tornados inúteis, seja pelo desgaste físico ou econômico. É computada por meio da seguinte expressão, proposta por Reis (1999): Depreciação $=$ [valor do recurso novo - valor residual do recurso (sucata)]/vida útil do recurso.

Nesta estimativa adotou-se, por convenção, a vida útil para benfeitorias de 20 anos e para máquinas e equipamentos, dez anos (Reis, 1999). Quanto ao valor residual dos equipamentos, considerou-se o valor final do bem após ser utilizado durante sua vida útil, ou seja, o seu valor de sucata. Segundo Reis (2002), na estimativa do valor de sucata pode-se utilizar um porcentual sobre o valor de um recurso novo, a exemplo de $10 \%$, estimando-se assim o valor final do bem, após ser utilizado de forma racional na atividade. Nesta pesquisa, o valor residual foi levantado por meio de informações dos fabricantes dos equipamentos, não representando necessariamente um porcentual fixo para cada item.

O custo alternativo ou de oportunidade (CA) é o retorno normal do capital empregado na atividade, ou seja, 
o que seria proporcionado se fosse aplicado em outra alternativa econômica. O custo fixo alternativo (CFAlt) foi calculado considerando-se como base a taxa de juros real esperada em aplicações da caderneta de poupança (6\% a.a.) pela seguinte expressão, conforme Reis (1999):

CFAlt $=[($ vida útil - idade média de uso do recurso $) /$ vida útil] x valor atual $\mathrm{x}$ taxa de juros. Consideraramse como componentes do custo fixo as instalações, equipamentos e instrumentos, materiais e remuneração das instalações. A instalação para a criação dos insetos deve ser composta de: uma sala de preparação $\left(4 \mathrm{~m}^{2}\right)$ com mesa, refrigerador e balança de precisão, onde são manuseados e armazenados os materiais relativos à produção de $O$. insidiosus, como os procedimentos de preparo do alimento (pesagem dos ovos de A. kuehniella), do substrato de oviposição e de limpeza dos recipientes de criação; uma sala de criação $\left(6 \mathrm{~m}^{2}\right)$ com oito prateleiras de aço $(30$ x95 cm), que comportam os recipientes de criação contendo os insetos predadores; e um canteiro de picão-preto (Bidens pilosa Linnaeu), que serviu de substrato para oviposição, com depreciação em nove ciclos de produção do predador.

Custo variável (CV) consiste nos recursos com duração inferior a um ciclo de produção, obtido pelo somatório de insumos, mão-de-obra e custo alternativo. Este custo foi estimado pelo desembolso na aquisição de produtos e serviços, acrescido dos respectivos custos alternativos. Quando não existe produção, não há custo variável. Assim, o custo variável alternativo (CVAlt) é igual ao subtotal do custo variável dividido por 2 vezes a taxa de juros, considerando-se que o capital variável é aplicado parceladamente no período de análise.

Foram considerados como componentes do custo variável os insumos gastos na criação do predador O. insidiosus, a mão-de-obra de um funcionário e os respectivos encargos sociais, assim como os honorários do responsável técnico. Além dos indicadores econômicos de custo, foi utilizada a análise do custo operacional (Cop), que se refere ao custo de todos os recursos de produção que exigem desembolso por parte da empresa, para sua recomposição. Inclui praticamente todos os recursos fixos que exigem reposição por meio de aquisições, sem considerar, no entanto, os custos alternativos. A finalidade do custo operacional é a opção de decisão nos casos em que retornos financeiros sejam inferiores aos de outras alternativas representadas pelo custo de oportunidade (Reis, 1999).

O custo total médio (CTMe) de produção é a soma do custo fixo total (CFT) e custo variável total (CVT) dividida pela quantidade produzida do predador, obtendo-se o custo de cada inseto.

Na análise econômica simplificada da atividade, utilizaram-se as situações descritas por Reis (2002), dependendo do preço ou receita média em relação aos custos (Figura 2). Neste estudo, considerou-se o preço

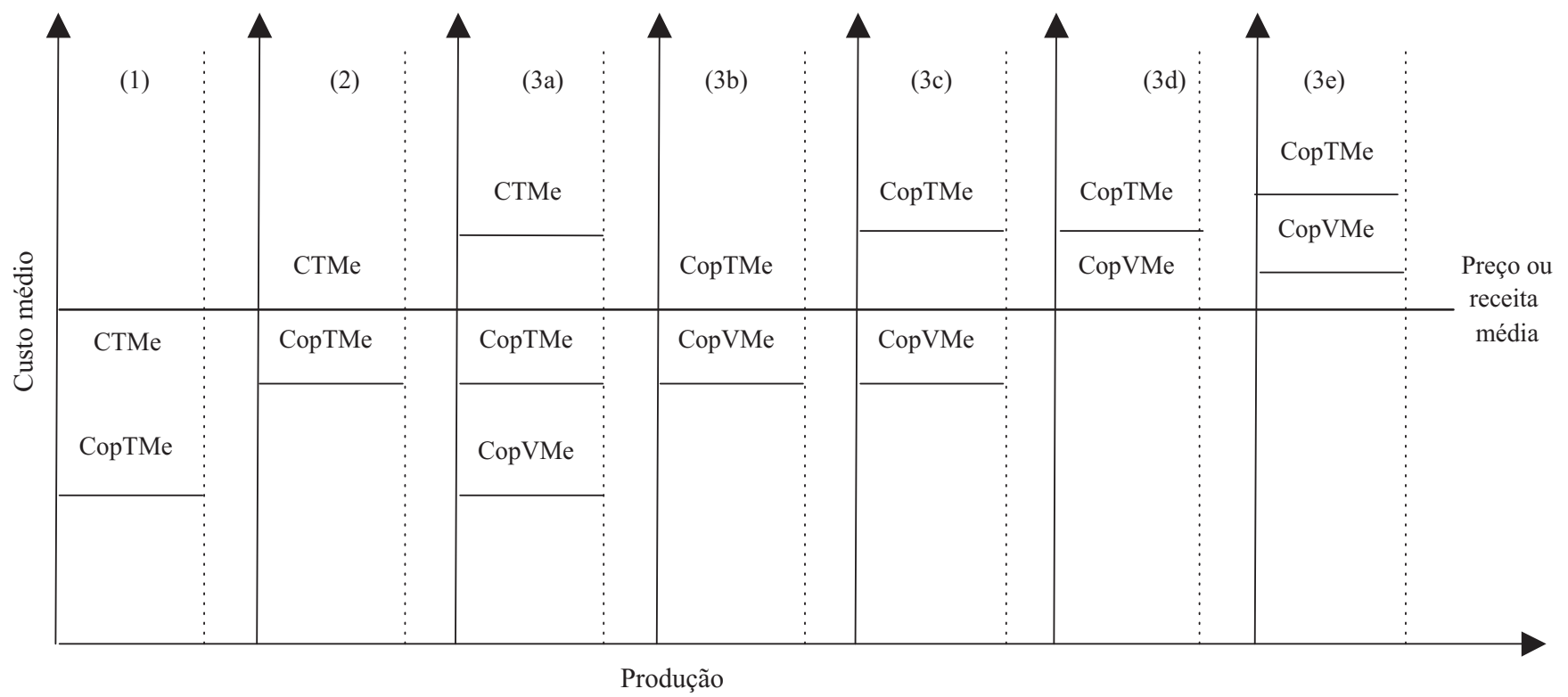

Figura 2. Situações de análises econômica e operacional de uma atividade produtiva com base no custo total médio (CTMe), custo operacional total médio (CopTMe) e custo operacional variável médio (CopVme), de acordo com Reis (2002). 
do produto e não a receita média da atividade, por não haver produção secundária ou subproduto. Esta análise poderá subsidiar o produtor a atribuir preço ao predador O. insidiosus produzido e auxiliar na escolha da atividade de criação desse inseto.

A situação 1 corresponde ao lucro supernormal (receita médica > CTMe), em que todos os recursos aplicados na atividade econômica são pagos e a atividade ainda proporciona um lucro adicional, superior ao de outras alternativas de mercado (custo alternativo). A receita média (RMe) pode ser considerada como o preço do produto mais o valor médio das vendas de explorações secundárias (subprodutos). Comparando-se a RMe ou o preço com os custos totais médios, obtémse a análise econômica da atividade em questão por unidade produtiva. Na situação 2, o lucro é considerado normal $(\mathrm{Rme}=\mathrm{CTMe})$; são pagos todos os recursos aplicados na atividade em questão e a remuneração é igual às alternativas do mercado, ou seja, o custo de oportunidade. A situação 3 , em que a rentabilidade da atividade é pior do que as remunerações do mercado, subdivide-se em cinco tipos: se a RMe > CopTMe, paga todos os recursos aplicados na atividade, com remuneração menor que a alternativa de mercado (3a); se a $\mathrm{RMe}=$ CopTMe, paga todos os recursos, porém, não paga o recurso alternativo (3b); se a RMe $>$ CopVMe, paga todos recursos variáveis e parte dos fixos (3c); se a $\mathrm{RMe}=\mathrm{CopVMe}$, paga somente os recursos variá- veis (3d); e se RMe < CopVMe não cobre o custo variável (3e).

\section{Resultados e Discussão}

Os resultados referentes aos custos da produção de Orius insidiosus estão apresentados nas planilhas de estimação de custo fixo total (Tabela 1) e de custo variável total (Tabela 2).

A estimativa do custo de produção do predador O. insidiosus em um sistema de criação em laboratório foi de US\$ 0,069 inseto-1 $^{-1}(\mathrm{R} \$ 0,197)$. Esse resultado foi alcançado mediante a divisão do custo total (R \$ 5.577,93), obtido a partir da soma do custo fixo total e do custo variável total ( $\mathrm{R}$ \$534,71 + R \$5.043,21), pelo número de indivíduos (28.272) produzidos na criação de O. insidiosus. Este valor refere-se à criação de $O$. insidiosus com lucros normais, os quais incluem todos os custos fixos e variáveis além da remuneração alternativa, ou seja, seu custo alternativo de emprego do capital ou custo de oportunidade. O lucro normal ocorre quando a receita for igual ao custo, ou seja, o preço recebido pelo produto se iguala ao seu custo total médio, quando neste se incluem os custos alternativos, que correspondem ao rendimento normal do capital e trabalho empregados no processo produtivo (Figura 2).

Tabela 1. Custo fixo discriminado da criação de Orius insidiosus (US\$1,00 = R \$2,84).

\begin{tabular}{|c|c|c|c|c|c|c|}
\hline Recursos & Valor $(\mathrm{R} \$)$ & $\begin{array}{c}\text { Vida útil } \\
\text { (ciclos de produção) }\end{array}$ & $\begin{array}{c}\text { Valor } \\
\text { residual }^{(1)}\end{array}$ & Depreciação & $\begin{array}{c}\text { Custo } \\
\text { alternativo }^{(2)}\end{array}$ & Custo fixo $^{(3)}$ \\
\hline Construção & $5.000,00$ & 90 & - & 55,56 & 160,00 & 215,56 \\
\hline Ar-condicionado & $1.100,00$ & 60 & 200,00 & 15,00 & 22,00 & 37,00 \\
\hline Refrigerador & 600,00 & 60 & 100,00 & 8,33 & 12,00 & 20,33 \\
\hline Estante de aço & 640,00 & 60 & 100,00 & 9,00 & 12,80 & 21,80 \\
\hline Balança & $1.711,00$ & 60 & 100,00 & 26,85 & 34,22 & 61,07 \\
\hline Microscópio & $2.000,00$ & 60 & 200,00 & 30,00 & 40,00 & 70,00 \\
\hline Mesa & 60,00 & 60 & 10,00 & 0,83 & 1,20 & 2,03 \\
\hline "Timer" & 100,00 & 60 & - & 1,67 & 2,00 & 3,67 \\
\hline Sugador portátil & 20,00 & 12 & - & 1,67 & 0,40 & 2,07 \\
\hline Pote de vidro & $1.106,00$ & 60 & 100,00 & 16,77 & 22,12 & 38,89 \\
\hline Pincéis, pinça, lápis, papel & 40,00 & 12 & - & 3,33 & 0,80 & 4,13 \\
\hline Papelão corrugado & 100,00 & 2 & - & 50,00 & 2,00 & 52,00 \\
\hline Piceta & 2,16 & 12 & - & 0,18 & 0,04 & 0,22 \\
\hline Total & - & - & - & 223,84 & 310,87 & 534,71 \\
\hline
\end{tabular}

(1)Valor desses equipamentos após a vida útil. (2)Considerou-se o valor do aluguel da sala (R\$ 80,00 mês-1) e dos demais equipamentos (o valor do novo vezes a taxa de juros real da caderneta de poupança). ${ }^{(3)}$ CopFT (custo operacional fixo total) $=\Sigma$ depreciações $=$ R\$ 223,84; Calt FT (custo alternativo fixo total) $=\Sigma$ custos alternativos $=\mathrm{R} \$ 310,87$; CFT (custo fixo total) $=\Sigma$ CopFT $+\Sigma$ custos alternativos $=$ R\$ 534,71; CFTMe (custo fixo total médio) $=\mathrm{R} \$ 534,71 / 28.272=\mathrm{R} \$ 0,0189$. 
Uma vez que não existem concorrentes no mercado nacional e o preço pode ser estabelecido pelo produtor do inseto, a comercialização pode ocorrer também com o preço fixado acima do custo de produção (preço > CTMe). Nesta situação, a atividade de produção de $O$. insidiosus alcançaria lucros supernormais, sugerindo condições de expansão, atraindo, assim, concorrentes para esse setor (Figura 2).

Crashaw et al. (1996) relataram que adultos do gênero Orius são comercializados nos EUA, em média, por US\$ 0,17, variando entre US\$ 0,10 e US\$ 0,28. As espécies mais comumente comercializadas são Orius tristicolor (White) e O. insidiosus. De acordo com esses autores, a recomendação do uso desses insetos como agente de controle varia entre 1.000 e 4.000 predadores ha-1 ${ }^{-1}$ dependendo da cultura e da intensidade do ataque da praga.

Dessa forma, o custo estimado de $O$. insidiosus se encontra abaixo do limite inferior do preço mencionado por Crashaw et al. (1996) nos EUA, podendo-se inferir que a metodologia de criação de $O$. insidiosus é considerada rentável ainda que adaptações devam ser feitas no intuito de melhorar a sua eficácia e os custos envolvidos no processo.

Por sua vez, o custo estimado do percevejo neste trabalho (US\$ 0,069) foi maior que o relatado por Schmidt et al. (1995), de US\$ 0,03. Entretanto, o valor encontrado por esse autor é relativo à criação de 50.000 a 100.000 indivíduos por semana. A criação do inseto em grande quantidade pode otimizar o sistema de criação, levando a ganhos de escala, ou seja, redução do custo por unidade produzida. Lenteren et al. (1997) mencionaram que $O$. insidiosus é produzido por quatro companhias diferentes na Europa e o preço mencionado por uma delas é de US\$ 0,04 predador $^{-1}$, também inferior ao encontrado neste estudo. Esses mesmos autores também citaram que as espécies Orius laevigatus (Fieber) e Orius majusculus (Reuter) são produzidas e comercializadas por cinco companhias, ao custo médio

Tabela 2. Custo variável discriminado da criação de Orius insidiosus (US\$ 1,00 = R\$2,84) ${ }^{(1)}$.

\begin{tabular}{|c|c|c|}
\hline Recurso & Quantidade & Valor $^{(2)}$ \\
\hline Salários e encargos sociais & 1 funcionário & $1.400,00$ \\
\hline Energia elétrica & - & 334,00 \\
\hline Algodão & $900 \mathrm{~g}$ & 20,00 \\
\hline Ovos de A. kuehniella & $292 \mathrm{~g}$ & 829,28 \\
\hline Material de limpeza & - & 10,00 \\
\hline Honorários & - & $2.400,00$ \\
\hline Custo operacional variável total (CopVT) & - & $4.993,28$ \\
\hline
\end{tabular}

de US\$ 0,072 e de US\$0,087, respectivamente. A relação custo/indivíduo deste predador indica que vários fatores, como a espécie de Orius e a quantidade de indivíduos produzidos, influenciam o custo final de produção.

Assim, a análise da atividade econômica por meio do custo de produção é um forte subsídio a decisões envolvendo atividade empresarial, apesar das dificuldades que normalmente ocorrem no processo de apuração dos dados e a subjetividade na sua estimação.

Lenteren et al. (1997) mencionaram que a recomendação de liberação desses predadores é de 1 a 10 Orius sp. $\mathrm{m}^{-2}$, dependendo da intensidade do ataque da praga. Silveira (2003) utilizou a densidade de 1,5 a 2,0 O. insidiosus $\mathrm{m}^{-2}$ no controle de tripes em crisântemo de corte produzido em sistema de cultivo protegido. No entanto, não existem estudos conclusivos sobre o número e o momento mais adequado para a liberação do predador, bem como a determinação dos níveis de controle das diferentes pragas às quais está associado, de modo a auxiliar decisões sobre uso. Assim, variáveis como tipo de cultura, método de cultivo e outros fatores (bióticos e abióticos) precisam ser estudadas para potencializar o efeito da liberação desses predadores no campo. Os níveis técnicos e econômicos de aceitação da praga na cultura também devem ser considerados.

A comercialização de inimigos naturais inclui o desafio da redução do custo de produção (Tauber et al., 2000). Alguns fatores, como a baixa viabilidade da criação na fase imatura de $O$. insidiosus, também precisam ser estudados, bem como a realização de ajustes metodológicos para melhorar a viabilidade e reduzir custos, permitindo a produção do predador de forma competitiva em biofábricas.

Cerca de $90 \%$ do custo total foram gastos com custo variável, e os gastos com honorários e mão-de-obra representam cerca de $62 \%$ do custo da criação desse inseto. Segundo Parra (2002), mesmo no Brasil, gastos com mão-de-obra representam entre $60 \%$ e $80 \%$ do custo de produção do inseto.

De acordo com o custo estimado para a produção de O. insidiosus (US\$ 0,069 inseto ${ }^{-1}$ ), e baseando-se no preço de comercialização praticado em outras partes do mundo (US\$ 0,03 a US\$ 0,28), os resultados encontrados sugerem a viabilidade econômica desta atividade. No entanto, há necessidade de se estabelecer a época e 
quantidade adequada para a liberação, de acordo com a praga-alvo, a cultura e os sistemas de cultivos a serem adotados. Segundo Parra (2002), é importante lembrar que, à medida que cresce o número de insetos criados, aumentam os problemas de instalações, sanidade, e qualidade do inimigo natural produzido. De acordo com esse autor, é fundamental que as atividades de produção e comercialização de inimigos naturais mantenham o respaldo técnico para ter credibilidade junto aos clientes e à comunidade, que ainda têm pouco acesso a essa forma de controle de pragas.

Assim, o custo de produção de $O$. insidiosus possibilita sua criação em laboratório e seu emprego como agente de controle em programas de controle biológico, principalmente de tripes, sua presa preferencial.

\section{Conclusão}

A estimativa do custo total médio de produção do predador Orius insidiosus criado em pequena escala é de R\$ 0,197 ou US\$ 0,069.

\section{Agradecimentos}

Ao CNPq e à Capes, pelas bolsas concedias aos autores; à Fapemig, pelo apoio financeiro.

\section{Referências}

BUENO, V.H.P. Desenvolvimento e multiplicação de percevejos predadores do gênero Orius Wolff. In: Controle biológico de pragas: produção massal e controle de qualidade. Lavras: Ufla, 2000. p.69-90.

CHAMBERS, D.L. Quality control in mass rearing. Annual Review of Entomology, v.22, p.289-308, 1977.

COHEN A.C.; NORDLUND, D.A.; SMITH, R.A. Mass rearing of entomophagous insects and predaceous mites: are the bottlenecks biological, engineering, economic, or cultural? Biocontrol News and Information, v.20, p.85-90, 1999.

CRANSHAW, W.; SCLAR, D.C.; COOPER, D. A review of 1994 pricing and marketing by suppliers of organisms for biological control of thrips in United States. Biological Control, v.6, p.291-296, 1996.

LENTEREN, J.C. Critérios de seleção de inimigos naturais a serem usados em programas de controle biológico. In: BUENO, V.H.P. (Ed.). Controle biológico de pragas: produção massal e controle de qualidade. Lavras: Ufla, 2000a. p.1-19.

LENTEREN, J.C. Greenhouse without pesticides: fact or fantasy? Crop Protection, v.19, p.375-384. 2000b.

LENTEREN, J.C.; ROSCAN, M.M.; TIMMER, R. Commercial mass production and pricing of organisms for biological control of pests in Europe. Biological Control, v.10, p.143-149, 1997.

LOPES, M.A.; CARVALHO, F. de M. Custo de produção de Leite. Boletim Agropecuário, v.33, p.1-17, 2000.

PARRA, J.R.P. Comercialização de inimigos naturais no Brasil, uma área emergente. In: PARRA, J.R.P.; BOTELHO, P.S.M.; CORREAFERREIRA, B.S.; BENTO, J.M.S. (Ed.). Controle biológico no Brasil: parasitóides e predadores. São Paulo: Ed. Manole, 2002. p.343-347.

REIS, R.P. Fundamentos de economia aplicada. 2002. 95p. Monografia (Especialização) - Universidade Federal de Lavras, Lavras, 2002.

SCHMIDT, J.M.; RICHARDS, P.C.; NADEL, H.; FERGUSON, G. A rearing method for the production of large numbers of the insidious flower bug, Orius insidious (Say) (Hemiptera: Anthocoridae). The Canadian Entomologist, v.127, p.445-447, 1995.

SILVEIRA, L.C.P. Registro e associação de espécies de Orius Wolff com tripes, influência do fotoperíodo na reprodução e avaliação de Orius insidiosus (Say, 1932) (Hemiptera: Anthocoridae) no controle biológico de tripes (Thysanoptera) em casa-de-vegetação. 2003. 104p. Tese (Doutorado) - Universidade Federal de Lavras, Lavras.

TAUBER, M.J.; TAUBER, C.; DAANE, K.M.; HAGGEN, K.S. Commercialization of predators: recent lessons from green lacewing (Neuroptera: Chrysopidae). American Entomologist, v.26, p.2638, 2000.

Recebido em 31 de maio de 2004 e aprovado em 7 de dezembro de 2004 